4. Libeau G, Diallo A, Parida S. Evolutionary genetics underlying the spread of peste des petits ruminants virus. Animal Frontiers. 2014;4:14-20. http://dx.doi.org/10.2527/af.2014-0003

5. Kock RA, Orynbayev MB, Sultankulova KT, Strochkov VM, Omarova ZD, Shalgynbayev EK, et al. Detection and genetic characterization of lineage IV peste des petits ruminant virus in Kazakhstan. Transbound Emerg Dis. 2015;62:470-9. http://dx.doi.org/10.1111/tbed.12398

6. International Union for Conservation of Nature and Natural Resources. The IUCN red list of threatened species. Capra aegagrus [cited $2016 \mathrm{Jul}$ 15]. http://dx.doi.org/10.2305/IUCN. UK.2008.RLTS.T3786A10076632.en

7. International Union for Conservation of Nature and Natural Resources. The IUCN red list of threatened species. Ovis orientalis [cited 2016 Jul 15]. http://dx.doi.org/10.2305/IUCN.UK.2008. RLTS.T15739A5076068.en

8. Zhu Z, Zhang X, Adili G, Huang J, Du X, Zhang X, et al. Genetic characterization of a novel mutant of peste des petits ruminants virus isolated from Capra ibex in China during 2015. BioMed Res Int. 2016;2016:7632769. http://dx.doi.org/10.1155/2016/7632769

9. Hoffmann B, Wiesner H, Maltzan J, Mustefa R, Eschbaumer M, Arif FA, et al. Fatalities in wild goats in Kurdistan associated with Peste des Petits Ruminants virus. Transbound Emerg Dis. 2012; 59:173-6. http://dx.doi.org/10.1111/j.1865-1682.2011.01270.x

10. Bailey D, Banyard A, Dash P, Ozkul A, Barrett T. Full genome sequence of peste des petits ruminants virus, a member of the Morbillivirus genus. Virus Res. 2005;110:119-24. http://dx.doi.org/10.1016/j.virusres.2005.01.013

Address for correspondence: Sasan Fereidouni, Conservation Medicine, Research Institute of Wildlife Ecology University of Veterinary

Medicine Vienna, Savoyenstrasse 1, Vienna, Austria;

email: sasan.fereidouni@vetmeduni.ac.at

\section{Discussion of Average versus Extreme Case Severity in Pandemic Risk Communications}

\section{Brian J. Zikmund-Fisher, Aaron M. Scherer, Megan Knaus, Enny Das, Angela Fagerlin}

Author affiliations: University of Michigan, Ann Arbor, Michigan, USA (B.J. Zikmund-Fisher, A.M. Scherer, M. Knaus); University of lowa, lowa City, lowa, USA (A.M. Scherer); Radboud University Nijmegen, Nijmegen, The Netherlands (E. Das); University of Utah, Salt Lake City, Utah, USA (A. Fagerlin); Salt Lake City VA Center for Informatics Decision Enhancement and Surveillance, Salt Lake City (A. Fagerlin)

DOI: http://dx.doi.org/10.3201/eid2304.161600

To investigate determinants of the public's perceptions of disease threat, in 2015 we conducted a randomized survey experiment in the Netherlands. Adults who read a mock news article describing average or extreme outcomes from a hypothetical influenza pandemic were more influenced by average than by extreme case information. Presenting both types of information simultaneously appeared counterproductive.

$\mathrm{W}$ hen pandemics strike, clear and timely communication is essential to raising public awareness of disease threat and motivating preventive behaviors (1). Yet, in most pandemics, the experience of affected persons is heterogeneous: a subset of persons have severe symptoms or sequelae, whereas most affected persons have much milder symptoms or sequelae. This heterogeneity creates a dilemma: Should communications about new infectious disease threats emphasize the character and severity of modal cases, which represents what most persons will experience, or should they focus on the severity of extreme cases to make clear the potential threat, even if that threat is highly unlikely? Both types of information are clearly important. Yet, risk messages are inherently difficult to understand, and providing multiple types of information simultaneously might undermine the public's understanding of a threat. Simplicity of message enables communications to stick with target audiences, and limiting communications to fewer, clearly contextualized, issues can increase efficacy $(2,3)$.

To begin to address this communications dilemma, during 2015 we conducted a randomized survey experiment with adult residents of the Netherlands who participate in an online panel administered by Survey Sampling International (https://www.surveysampling.com/). We established quotas for age and sex that approximated the distributions of these characteristics in the population of the Netherlands (online Technical Appendix, https://wwwnc.cdc.gov/EID/ article/23/4/16-1600-Techapp1.pdf). Upon completing the survey, participants received modest prizes.

Participants read a mock news article about a new pandemic (referred to as H7N3 influenza) spreading within the Netherlands. We randomly varied how the article discussed the average case severity, which was 1) not discussed, 2) described as mild (moderate fever and cough; generally goes away by itself), or 3) described as moderately severe (high fever, cough, vomiting; generally requires intravenous medication and hospitalization). We also independently varied the description of extreme cases, which were 1) not discussed, 2) described as (relatively) mild (requiring 1-2 days of hospitalization because of difficulty breathing, dizziness, and persistent coughing), or 3) described as moderately severe (requiring hospitalization [and causing 1 death] because of difficulty breathing, dizziness, severe coughing, and fluid in the lungs). This randomization resulted in a $3 \times 3$ between-subjects factorial design. Following guidelines for effective health messages (4), all articles 
Table. Differences in ratings of worry and vaccination intentions compared with ratings when no information was provided about a hypothetical influenza pandemic, the Netherlands, 2015*

\begin{tabular}{lccc}
\hline & & Extreme case scenario & Moderate severity \\
\cline { 2 - 4 } Average case scenario & No Information & Mild severity & +0.20 \\
\hline No information & Reference & -0.01 & +0.13 \\
$\quad$ Worry if symptoms & Reference & +0.07 & +0.18 \\
$\quad$ Worry about extreme & Reference & -0.16 & -0.07 \\
$\quad$ Vaccination intentions & & & -0.08 \\
\hline Mild severity & -0.23 & -0.01 & +0.01 \\
$\quad$ Worry if symptoms & -0.07 & -0.18 & -0.12 \\
$\quad$ Worry about extreme & -0.25 & & +0.17 \\
$\quad$ Vaccination intentions & & +0.18 & +0.13 \\
\hline Moderate severity & +0.26 & +0.13 & -0.06 \\
$\quad$ Worry if symptoms & +0.22 & +0.05 & \\
$\quad$ Worry about extreme & +0.21 & & \\
\hline Vaccination intentions & & & \\
\hline+ increased worry; - , decreased worry. & & & \\
\hline
\end{tabular}

included a (fixed) efficacy message, instructing readers to cover their mouths for coughs and sneezes and wash hands frequently to prevent disease spread (online Technical Appendix). This design received exempt status approval from the University of Michigan Medical Institutional Review Board (Ann Arbor, MI, USA).

Our analyses focused on 3 questions: how much respondents would worry if symptoms developed, how much they would worry about extreme effects if they contracted the disease, and participants' vaccination intentions if a vaccine were available. All questions were 5-point Likert scales, where higher values represented greater worry or intent to vaccinate. Although absolute rates of concern and vaccination intentions are not generalizable from the hypothetical scenario, significant differences among the experimental conditions should be. We conducted $3 \times 3$ analyses of variance and ordered logistic regression analyses of each outcome with variables for each level of average and/or extreme case information (not present, mild, moderate). The results showed close correspondence, so for simplicity we report only analysis of variance results.

A total of 2,695 participants completed the survey and answered the 3 primary outcome questions. Average age was 49.2 ( $\mathrm{SD} \pm 15.6$; range $18-96$ ) years, and $49.8 \%$ of respondents reported being female.

Overall, respondents were most sensitive to descriptions of average case severity: worry if symptoms: $\mathrm{F}(2,2686)=20.87, \quad \mathrm{p}<0.001$; worry about extreme: $\mathrm{F}(2,2686)=6.16, \mathrm{p}=0.002$; vaccination intentions: $F(2,2686)=7.56, p<0.001$. By contrast, the main effect of extreme case information was nonsignificant in all 3 analyses $(0.16<p<0.77)$. However, we noticed evidence of an interaction effect for vaccination intentions $(\mathrm{F}[2,2686]=3.23, \mathrm{p}=0.01)$.

The main effect of average case information was clearly visible among respondents receiving no information about extreme cases (Table, first column). Yet, the effect of average case information appears muted (less variance) when extreme case descriptions were also presented. In fact, if participants were told that the average case was moderately severe (Table, bottom row), adding extreme case information (either severity level) did not increase worry or vaccination intentions, and the trend is negative.

Our data suggest that information about average cases and extreme cases did not have additive effects on participants' responses. We observed the strongest effects (positive and negative) of average case information when information about extreme cases was not provided. Providing average case information might inhibit consideration of just how serious the disease could be. Average case information also might have higher personal relevance to the public because extreme cases are more easily discounted. If so, public health communications about new threats should avoid presenting both types of information simultaneously.

Funding for this research was provided to A.F. from the European Union's Seventh Framework Programme for research, technological development, and demonstration under grant agreement \#278763. The funding agreement ensured the authors' independence in designing the study, in the collection, analysis, and reporting of the data, and in the decision to submit the article for publication.

Dr. Zikmund-Fisher is associate professor of Health Behavior and Health Education at the University of Michigan School of Public Health. His research focuses on determinants of health risk perceptions and designing effective health risk communications.

\section{References}

1. Bults M, Beaujean DJMA, Richardus JH, Voeten HACM. Perceptions and behavioral responses of the general public during the 2009 influenza A (H1N1) pandemic: a systematic review. Disaster Med Public Health Prep. 2015;9:207-19. http://dx.doi.org/10.1017/dmp.2014.160

2. Peters E, Dieckmann N, Dixon A, Hibbard JH, Mertz CK. Less is more in presenting quality information to consumers. Med Care Res Rev. 2007;64:169-90. http://dx.doi.org/10.1177/107755870706 40020301

3. Zikmund-Fisher BJ. The right tool is what they need, not what we have: a taxonomy of appropriate levels of precision in patient risk 
communication. Med Care Res Rev. 2013;70(Suppl):37S-49S. http://dx.doi.org/10.1177/1077558712458541

4. Peters G-JY, Ruiter RAC, Kok G. Threatening communication: a critical re-analysis and a revised meta-analytic test of fear appeal theory. Health Psychol Rev. 2013;7(Suppl 1):S8-31. http://dx.doi.org/10.1080/17437199.2012.703527

Address for correspondence: Brian J. Zikmund-Fisher, Department of Health Behavior and Health Education, University of Michigan, 1415 Washington Heights, Ann Arbor, MI 48109-2029, USA; email: bzikmund@umich.edu

\section{West Nile Virus Seroprevalence, Connecticut, USA, 2000-2014}

\author{
Megan E. Cahill, Yi Yao, David Nock, \\ Philip M. Armstrong, Theodore G. Andreadis, \\ Maria A. Diuk-Wasser, Ruth R. Montgomery
}

Author Affiliations: Yale University School of Public Health, New Haven, Connecticut, USA (M.E. Cahill); Yale University School of Medicine, New Haven (Y. Yao, D. Nock, R.R. Montgomery); The Connecticut Agricultural Experiment Station, New Haven (P.M. Armstrong, T.G. Andreadis); Columbia University, New York, New York, USA (M.A. Diuk-Wasser)

DOI: http://dx.doi.org/10.3201/eid2304.161669

West Nile virus (WNV) infection is mainly asymptomatic but can be severe in elderly persons. As part of studies on immunity and aging in Connecticut, USA, we detected WNV seroconversion in $8.5 \%$ of nonimmunosuppressed and $16.8 \%$ of immunosuppressed persons. Age was not a significant seroconversion factor. Our findings suggest that immune factors affect seroconversion.

Since the 1999 emergence of West Nile virus (WNV) in North America, $>43,000$ cases of disease and 1,884 deaths have been reported $(1)$; overall infections are estimated at $\approx 3$ million (2). Although WNV infections can be asymptomatic, they can also cause severe neuroinvasive disease, especially among infants, immunocompromised persons, and elderly persons (3). Control of WNV infection involves innate immune pathways that mediate initial recognition and regulation of viral replication and adaptive immune responses that provide long-term protection (3). Spatial distribution analysis and mosquito surveillance studies have confirmed that WNV is endemic to Connecticut, USA $(1,4)$.
We compared seroprevalence and demographics for 890 nonimmunosuppressed and 173 immunosuppressed adults enrolled in a study on immunity in aging (approved by the Human Investigations Committee of Yale University) (5) with those of symptomatic WNV case-patients reported to the Connecticut Department of Health (DPH) during 2000-2014. DPH-reported symptomatic case-patients $(\mathrm{n}=116)$ sought medical attention and had a positive WNV laboratory test result (1). None of the asymptomatic participants were reported to DPH as WNV case-patients. Immunosuppressed participants followed an immunosuppressive medication regimen or had a diagnosis of rheumatoid arthritis (5). For all participants, we assessed previous exposure to WNV by immunoblot for WNV envelope protein (6). Seroconversion to WNV was distinguished from cross-reactivity to other flaviviruses by rescreening all positive serum against a recombinant WNV-specific mutant envelope protein that lacks the conserved cross-reactive fusion loop epitope (7).

We compared demographic characteristics of participant groups by using the Student $t$-test for continuous variables and $\chi^{2}$ and Fisher exact tests for categorical variables; $\mathrm{p}<0.05$ indicated statistical significance. Analysis was completed with SAS software version 9.3 (SAS Institute, Cary, NC, USA) and Prism 6 (GraphPad Software, Inc., La Jolla, CA, USA).

Immunoblot detected evidence of WNV exposure in $76(8.5 \%)$ of the 890 nonimmunosuppressed participants (Table). These seropositive participants reported neither symptoms nor diagnosis of WNV infection and are considered to have had asymptomatic infections. Timing of asymptomatic infections could not be determined, but antibodies against $\mathrm{WNV}$ are durable and do not differ between asymptomatic and symptomatic adults (8).

Although age is a critical risk factor for severe WNV infection $(3,9)$, the mean age of seropositive and seronegative nonimmunosuppressed participants did not differ significantly (Table). The rate of asymptomatic seroconversion did not vary significantly among the 890 persons in 3 age groups: $<35$ years $(42 / 421), 35-65$ years $(7 / 121)$, and $>65$ years $(27 / 348)(p=0.338)$. Seroconversion rates did not differ significantly by patient sex but were significantly elevated among those in self-identified Hispanic groups $(p<0.0001)$, possibly because of different exposure histories. The similar age distribution among asymptomatic seroconverters suggests that the observed age-associated susceptibility to clinically apparent disease may result from other factors, including individual host factors and dysregulation in immune responses $(6,10)$.

Among 173 immunosuppressed adults, 29 (16.8\%) showed evidence of exposure to WNV (Table), resulting in 2.16 times the odds of positive immunoblot result than for nonimmunosuppressed adults $(76 / 890,8.5 \% ; \mathrm{p}=0.002)$. 